\title{
Revisión bibliométrica sobre el estudio de la Teoría de la Mente y la Personalidad*
}

\section{Bibliometric review about Theory of Mind and Personality}

\author{
Hernán Darío Lotero Osorio** \\ ORCID: 0000000214323199 \\ Nora Helena Londoño Arredondo \\ ORCID: 0000000160161955 \\ Universidad de San Buenaventura, Medellín, \\ Colombia \\ Recibido: 7 de junio de 2018 \\ Revisado: 6 de septiembre de 2018 \\ Aceptado: 4 de diciembre de 2018
}

\section{Resumen}

El propósito del presente artículo es presentar una revisión bibliométrica sobre las investigaciones que trabajan el concepto de Teoría de la Mente (ToM) y de Personalidad. Se obtuvieron 75 artículos y se seleccionaron 30 estudios de las bases de datos Scopus, Science Direct, EBSCO, PubMed, Scielo y Springer, publicados entre los años 2012 y 2016. Se halló una relación de la ToM con trastornos de la personalidad, tales como el trastorno límite, narcisista y antisocial. También se encontró relación con rasgos específicos, como baja empatía, el tipo de apego y los altos niveles de insensibilidad emocional. Se concluye que, aunque se ha avanzado en el conocimiento de la relación entre la ToM y la personalidad, las investigaciones no son lo suficientemente extensas para aclarar cómo opera esta interacción con la totalidad de las dimensiones y los rasgos de personalidad en sujetos con y sin psicopatología.

Palabras clave: personalidad, teoría de la mente, revisión bibliométrica, trastornos de la personalidad.

\footnotetext{
Artículo de investigación. Grupo de investigación: Estudios clínicos y sociales en psicología. Cómo citar: Lotero, O. H. D. \& Londoño, A. N. H. (2019). Revisión bibliométrica sobre el estudio de la Teoría de la Mente y la Personalidad. Diversitas: Perspectivas en Psicología, 15(2), 231-249. DOI:10.15332/22563067.3906

** Correspondencia: Hernán Darío Lotero Osorio; Nora Helena Londoño Arredondo, Grupo de investigación estudios clínicos y sociales en psicología. Correo electrónico: nora.londono@usbmed.edu.co Dirección postal: Carrera 56C n. 51-110 (Barrio San Benito), Universidad de San Buenaventura, Medellín, Colombia.
} 


\section{Abstract}

The aim of this article is to present a bibliometric review on research that works on Theory of Mind (ToM) and Personality. Initially, we found 75 studies of which Thirty studies were selected from the Scopus, Science Direct, EBSCO, PubMed, Scielo and Springer databases, published between 2012 and 2016. We found a relationship between ToM with personality disorders such as, borderline, narcissistic and antisocial disorder. Also, a relationship with specific features such as low empathy, attachment type and high levels of emotional insensitivity. It is concluded that although progress has been made in the knowledge of the relationship between ToM and personality, the investigations are not extensive enough to clarify how this interaction operates with all dimensions and personality traits in subjects with and without psychopathology.

Keywords: personality, Theory of Mind, bibliometric review, personality disorders.

\section{Introducción}

La Teoría de la mente (ToM) es un término propuesto por los primatólogos Premack y Woodruff (1978) para un componente de la cognición social que hace referencia a la capacidad para realizar inferencias acerca de los estados mentales de otras personas, como por ejemplo intenciones, disposiciones, creencias y emociones (Brüne, 2005; Schenkel, Spaulding, \& Silverstein, 2005). La teoría de la mente supone hacer uso de creencias, deseos, intenciones, sentimientos, entre otros, para conocer, entender e interpretar el comportamiento de las personas. Es decir, la teoría de la mente hace referencia entonces a la habilidad para comprender y predecir la conducta de otras personas, sus conocimientos, sus intenciones y sus creencias (García, 2008).

La ToM en los seres humanos comenzó a trabajarse por parte de Baron-Cohen, Leslie y Frith (1985), y desde allí su desarrollo cronológico comenzó a ser investigado. En un primer momento la ToM fue estudiada con relación al desarrollo del lenguaje y a la expresión empática del mismo (Hood, Bloom, \& Brainerd, 1979; Shultz, Wells, \& Sarda, 1980) lo cual situó su desarrollo a partir de los 2 años de edad (Bretherton \& Beeghly, 1982). Otras investigaciones han retomado el concepto de historias de falsa creencia y lo han ubicado a la edad de 4 a 5 años, donde se comienza a comprender y predecir el comportamiento de los demás (Wimmer \& Perner, 1983). Sin embargo, otras investigacio- nes basadas en el Faux pas ("metedura de pata") (Happé, 1993) permitieron plantear su desarrollo entre los 3 a 4 años de edad, aunque diferencian estas falsas creencias de primer orden, a las falsas creencias de segundo orden entre 6 a 7 años de edad y las meteduras de patas, entendimiento de sinónimos, metáforas e ironías entre 9 a 11 años de edad (Stone, Baron-Cohen \& Knight, 1998). Este hallazgo sería posteriormente corroborado, pero refiriendo una diferencia entre sexos con predominancia del género femenino en términos de desarrollo de la ToM (Baron-Cohen, O'Riordan, Stone, Jones \& Plaisted, 1999).

Otra línea de investigación de la ToM ha pretendido la construcción y validación de instrumentos que midan esta habilidad. Entre estos se encuentran la historia de maxi y del chocolate para falsa creencia de primer y segundo orden (Wimmer \& Perner, 1983). El test de Sally y Ann para medir la falsa creencia de primer orden (Baron-Cohen et al., 1985). La historia del heladero para falsa creencia de segundo orden (Baron-Cohen, 1989). El test de reconocimiento de mentiras, mentiras piadosas, bromas, simulación, malentendidos, persuasión, apariencia / realidad, lenguaje figurativo, sarcasmo, olvidos, engaño doble y emociones contrarias (Happé, 1994). El Hinting Task para el entendimiento del lenguaje indirecto (Corcoran, Mercer, \& Frith, 1995). El test del cómic para la falsa creencia y el humor (Corcoran, Cahill, \& Firth, 1997). El test de la lectura de la mirada para medir el reconocimiento emocional a través de la región 
de los ojos (Baron-Cohen, Jolliffe, Mortimore \& Robertson, 1997). El Faux pas ("metedura de patas") para medir entendimiento de errores sociales (Stone et al., 1998). El ToM Test que mide diversos niveles de complejidad de esta habilidad, iniciando en el reconocimiento de emociones y juego simbólico, comprensión de la falsa creencia de primer orden y comprensión de la falsa creencia de segundo orden y del humor (Muris et al., 1999).

Así mismo, el test de historias cotidianas que mide las mentiras en la comunicación social, una mentira piadosa, discurso figurativo, malentendidos, un doble engaño, ironías, persuasiones, emociones contrarias, olvidos, celos, intenciones, empatía y errores sociales (Kaland et al., 2002). El test de las situaciones de metáforas y sarcasmo que mide el entendimiento de estos dos conceptos lingüísticos (Adachi et al., 2004). La escala de ToM que mide la comprensión de deseos distintos versus creencias distintas, el acceso al conocimiento versus la falsa creencia de primer orden, emociones ligadas a creencias y apariencias versus realidad emocional (Wellman, Fang, Liu, Zhu \& Liu, 2006; Wellman \& Liu, 2004). El NEPSY-II (A Developmental Neuropsychological Assessment-Second Edition) que permite la medición de la ToM en conjunto con otras funciones neurocognitivas como la capacidad atencional, funciones ejecutivas, lenguaje, memoria, aprendizaje, capacidades sensoriomotoras, procesamiento visoespacial y percepción social (Korkman, Kirk, \& Temp, 2007).

Finalmente, el libro e historias de La ToM, el cual incluye la evaluación de 5 áreas principales: reconocimiento de emociones, diferenciación entre entidades físicas y mentales, conocimiento obtenido por la percepción de los eventos, deseos y falsas creencias (Blijd-Hoogewys, van Geert, Serra, \& Minderaa, 2008). Y posteriormente, como método para facilitar el acercamiento, y promover mayores niveles de sensibilidad y de empatía con los personajes de los test empleados para la medición de la ToM, se crearon otros instrumentos que se encontraban basados en la proyección de videos a los participantes de la investigación. Entre estas pruebas destacan, el test del momento incómodo (Heavey, Phillips, Baron-Cohen, \& Rutter, 2000), el Empathic Accuracy Paradigm (Roeyers, Buysse, Ponnet, \&
Pichal, 2001) y el MASC Movie For Assesment of Social Cognition (Dziobek et al., 2006).

\section{Neurobiología de la ToM}

Se ha referido que el sistema nervioso dispone de mecanismos neuronales que se encuentran especializados para percibir, discriminar y reconocer los estados mentales. La combinación de estructuras relacionadas con la interpretación de aspectos afectivos y emocionales, con estructuras que permiten el uso de funciones ejecutivas e implicación de apropiación de la conducta son las que se han identificado en el proceso de la ToM. En la actualidad se reconocen la corteza prefrontal ventromedial, la amígdala, la corteza somatosensorial y la ínsula (Téllez-Vargas, 2006).

La corteza prefrontal muestra una relación con la ToM en lo referente al control voluntario del comportamiento, la conciencia y las funciones ejecutivas. Esta área presenta implicación durante el proceso de pensar como los demás, esto mediante el uso de la activación del lóbulo frontal, que le permite al sujeto separar la propia perspectiva de la de los demás (Marchetti et al., 2015).

La amígdala interviene en la cognición social en la regulación de los componentes emocionales en las diferentes situaciones sociales, desempeñando una importante función en la lectura y la relación de señales sociales adecuadas, las cuales están asociadas al funcionamiento del núcleo basal por ser este el de mayor interconexión con la corteza prefrontal ventromedial (Emery \& Amaral, 2000). Del mismo modo, los estudios realizados con lesiones en la amígdala han demostrado la importancia para el reconocimiento de expresiones faciales de emociones, especialmente del miedo, por lo que se puede afirmar que la amígdala desempeña una función crucial en el reconocimiento y la identificación de las emociones (Broks et al., 1998).

La corteza somatosensorial derecha y la ínsula se relacionan con la capacidad de detección de lo que otra persona siente (Zaborszky et al., 1997), y del reconocimiento de emociones sociales (Lamm \& Singer, 2010) respectivamente. Se ha reportado que esta capacidad de interpretación y asociación de la 
expresión emocional en un rostro con alguna de las emociones básicas esta mediada por estas estructuras (Adolphs et al., 1994). Por lo tanto, los pacientes con lesión en estas áreas presentan trastornos en el juicio emocional (Zaborszky et al., 1997).

En resumen, la corteza prefrontal ventromedial permite una integración entre la percepción de una emoción y la respuesta que desencadena, esta respuesta es mediada a su vez por la amígdala quien determina la acción adecuada. Y la corteza somatosensorial derecha y la ínsula permiten una correcta manipulación de la información para permitir la interpretación facial de la expresión emocional que presenta otra persona dando especial énfasis en su mirada.

\section{ToM y patología}

Actualmente se han realizado múltiples estudios, revisiones sistemáticas y metaanálisis que han concluido y hallado relación entre la ToM y algunas patologías (Martins-Junior, Sanvicente-Vieira, Grassi-Oliveira \& Brietzke, 2011). Sin embargo, estas enfermedades no son referidas únicamente a aspectos psicopatológicos, sino que además se han encontrado importantes hallazgos que relacionan la ToM con algunas otras condiciones patológicas de naturaleza fisiológica (Adenzato, Cavallo, \& Enrici, 2010; Bora, Velakoulis, \& Walterfang, 2016; Bora, Walterfang \& Velakoulis, 2015).

En relación con las condiciones de alteraciones mentales, diferentes estudios han establecido la relación entre la ToM con el autismo y el síndrome de Down, presentando una menor disfunción de la ToM en esta última condición En uno de estos metaanálisis se comparó a sujetos normales con un grupo de individuos diagnosticado con autismo y otro grupo de personas diagnosticado con retraso mental, se encontraron déficits en la capacidad de la ToM, tanto en el grupo con autismo como en el de retraso mental (Baker, Peterson, Pulos, \& Kirkland, 2014; Trevisan \& Birmingham, 2016; Yirmiya, et. al, 1998).

En otros metaanálisis se ha encontrado que el trastorno afectivo bipolar en su fase sintomática presenta déficits en los niveles de la ToM, y se encontró que mientras mayor sea el grado de intensidad en los síntomas se presentan menores niveles de ToM (Bora, Bartholomeusz, \& Pantelis, 2015). De igual manera se evidenció que dicha disfunción de la ToM también se presenta en episodios de remisión sintomática o en fase eutímica de este trastorno (Samamé, Martino, \& Strejilevich, 2011).

El Trastorno de Déficit de Atención por Hiperactividad (TDAH) también fue comparado en sus niveles de ToM con pacientes diagnosticados con Asperger y sujetos control, en el metaanálisis se concluyó que los pacientes con TDAH presentaban un puntaje intermedio de ToM entre los sujetos control y los pacientes que presentaban Asperger. Además, este estudio resaltó la importancia de la edad para el desarrollo de los niveles de la ToM en los sujetos con estas condiciones (Bora \& Pantelis, 2015).

En Esquizofrenia son múltiples los trabajos que han identificado afección en el área de la cognición social lo cual ha permitido realizar metaanálisis acerca del tema (Savla, Vella, Armstrong, Penn, \& Twamley, 2012). En otro estudio se estableció la disminución presentada de la ToM en pacientes con esquizofrenia, encontrando niveles más debilitados en esta habilidad que los sujetos control. Además, estos resultados se presentaron tanto en sujetos con síntomas activos como en aquellos con remisión de episodios psicóticos, aunque estos últimos presentaban una disfunción menos notoria que aquellos en fase activa (Bora, Yucel, \& Pantelis, 2009).

En este mismo sentido, en una comparación de los niveles de cognición social entre pacientes con esquizofrenia y otros con autismo (Bliksted, Ubukata, $\&$ Koelkebeck, 2016), se encontró que los diferentes niveles en los estados patológicos de la esquizofrenia demostraban a su vez diversos estados de déficits de cognición social, llevando con esto a hipotetizar una correlación entre ambos factores, demostrando mayores niveles de afectación de la ToM en presencia de mayores niveles de estados psicóticos activos. Asimismo, se ha mostrado que altos niveles de ToM en personas con esquizofrenia correlacionan con mejores grado de funcionalidad en estos pacientes (Fett et al, 2011). Otro aspecto relevante es que se han encontrado niveles bajos de ToM en los parientes en primera línea de 
consanguinidad de pacientes con esquizofrenia, llevando con esto a proponer una posible influencia de factores hereditarios en el desarrollo de la cognición social en pacientes con esquizofrenia (Bora \& Pantelis, 2013; Lavoie et al., 2013), aunque también se ha encontrado que en la presentación de los primeros síntomas de esquizofrenia no se encuentra deterioro en esta función, por lo cual el déficit suele asociarse al desarrollo de la patología y no a su génesis (van Donkersgoed, Wunderink, Nieboer, Aleman \& Pijnenborg, 2015).

En lo relacionado con patologías de orden físico, también se ha investigado los niveles de ToM en algunas de estas condiciones. En la enfermedad de Huntington se encontró deterioro en los niveles de cognición social, específicamente en la identificación emocional mediante estímulos verbales y faciales, además en el reconocimiento de emociones negativas como el asco, la ira y el miedo, llevando con esto a hipotetizar que la presencia de disfunción en la capacidad de la ToM puede corresponder a indicios de aparición de la enfermedad o a un avance en la misma (Bora, Velakoulis, \& Walterfang, 2016). En la demencia frontotemporal, más específicamente en su variante comportamental, se ha referido la presencia de alteraciones en el nivel de la ToM, encontrando bajos niveles en esta habilidad, demostrando con esto dificultades para la interpretación de estados mentales ajenos y por lo tanto dificultades de orden relacional en los pacientes con esta condición. Es por esto que mediante técnicas de neuroimagen se analizó el comportamiento de la actividad neuronal, y se encontró que en esta patología se hallaba una degeneración progresiva de las estructuras de la región anterior fronto-medial, la cual es la causante de la presentación de la demencia frontotemporal y simultáneamente de la capacidad disminuida de la ToM (Adenzato, et al., 2010).

\section{ToM y Personalidad}

En lo referente a los aspectos de la personalidad se han adelantado investigaciones desde el campo de los trastornos de personalidad. Hasta la actualidad se ha encontrado que el trastorno límite de personalidad ha mostrado niveles de ToM inadecuados, refiriendo aspecto de mentalizar de forma superlativa, causando errores por sobre interpretación de las conductas o intenciones humanas (Baez et al., 2014; O’Neill et al., 2015). En el trastorno esquizoide se presenta dificultad por infra-mentalización para interpretar emociones de tipo positivas (Deptula \& Bedwell, 2015; Henry, Bailey, \& Rendell, 2008). Y en el trastorno antisocial debido a la baja capacidad de reconocimiento emocional, y a la presencia de niveles inferiores de empatía con los demás individuos (Nentjes, Bernstein, Arntz, Slaats, \& Hannemann, 2015; Nentjes, Bernstein, Arntz, van Breukelen, \& Slaats, 2015; Stellwagen \& Kerig, 2013; Vonk, Zeigler-Hill, Ewing, Mercer, \& Noser, 2015).

Como se puede evidenciar, la información científica que relaciona las variables de la ToM y personalidad es limitada, motivo por el cual esta revisión busca contribuir en la síntesis de la evidencia científica disponible hasta la actualidad acerca de la ToM, y su actual estatus en relación al concepto de personalidad, lo cual permitirá identificar aspectos relacionados con la publicación de material científico y el panorama actual de la investigación sobre estas variables.

\section{Método}

El proceso de revisión implicó la selección de estudios clínicos acerca de la teoría de la mente, mediante búsqueda electrónica en las bases de datos Scopus, Science Direct, EBSCO, PubMed, Scielo y Springer, de los cuales se obtuvieron 75 artículos científicos. Se realizó una lectura detallada de títulos y resúmenes de trabajos publicados entre los años 2012 y 2016, para la determinación de cumplimiento de requisitos para su escogencia. Los criterios de búsqueda (en inglés) fueron: teoría de la mente ("theory of mind"), personalidad y teoría de la mente ("personality and theory of mind"), teoría de la mente y trastornos de personalidad ("theory of mind and persanality disorders") y teoría de la mente y rasgos de personalidad ("theory of mind and personality traits").

Los criterios de inclusión fueron: el tipo de diseño del estudio, especialmente estudios experimentales controlados; el tipo de instrumentos de medición utilizados; las variables de medición y los años de publicación de los resultados (2012-2016). La tabla 1 muestra los resultados de la búsqueda. 
Tabla 1.

Estrategia de búsqueda e identificación de estudios

\begin{tabular}{llc}
\hline Base de Datos & Términos de búsqueda & Número de artículos \\
\hline SCOPUS & $\begin{array}{l}\text { Personalidad y ToM, rasgos de personalidad y ToM, } \\
\text { trastornos de personalidad y ToM }\end{array}$ & 8 \\
\hline Springer & $\begin{array}{l}\text { Personalidad y ToM, rasgos de personalidad y ToM, } \\
\text { trastornos de personalidad y ToM }\end{array}$ & 6 \\
\hline PubMed Central -PMC- & $\begin{array}{l}\text { Personalidad y ToM, rasgos de personalidad y ToM, } \\
\text { trastornos de personalidad y ToM }\end{array}$ & 6 \\
\hline EBSCOhost & $\begin{array}{l}\text { Personalidad y ToM, rasgos de personalidad y ToM, } \\
\text { trastornos de personalidad y ToM }\end{array}$ & 5 \\
\hline Science Direct & $\begin{array}{l}\text { Personalidad y ToM, rasgos de personalidad y ToM, } \\
\text { trastornos de personalidad y ToM }\end{array}$ & 5 \\
\hline Total & & 30 \\
\hline
\end{tabular}

Fuente: elaboración propia

De la totalidad de los artículos rastreados (75), se identificaron y seleccionaron 30 estudios los cuales contenían la información científica relevante para los propósitos de esta revisión, estos fueron elegidos mediante la lectura de la totalidad del artículo y de la búsqueda del cumplimiento de los criterios de inclusión anteriormente descritos, procedimiento realizado por un estudiante de doctorado. De los 30 estudios seleccionados fueron extraídos los datos de número de artículos publicados por año, revistas de publicación de los artículos, autores, países de producción, principales áreas de estudio de las revistas indexadas, participantes, test empleados, las variables principales de estudio y principales resultados.

Los 30 artículos se analizaron sistemáticamente empleando el software SPSS24, y con este se realizó la exploración estadística descriptiva que permitió construir los análisis de la variable de personalidad estudiada en torno a la ToM.

Para el análisis de los resultados, se empleó una metodología cualitativa y cuantitativa, con el fin de identificar tanto aspectos descriptivos de la distribución estadística de los artículos incluidos en el estudio, así como los test empleados, las principales variables trabajadas y las principales conclusiones obtenidas en cada artículo.

\section{Resultados}

En la tabla 2 se presentan los artículos seleccionados para el actual estudio, así como sus respectivos autores, la población empleada y el test de ToM utilizados. Es importante mencionar que la muestra total de los estudios clínicos estuvo constituida por 5281 personas, de las cuales 4177 fueron adultos, 764 adolescentes y 340 niños. 


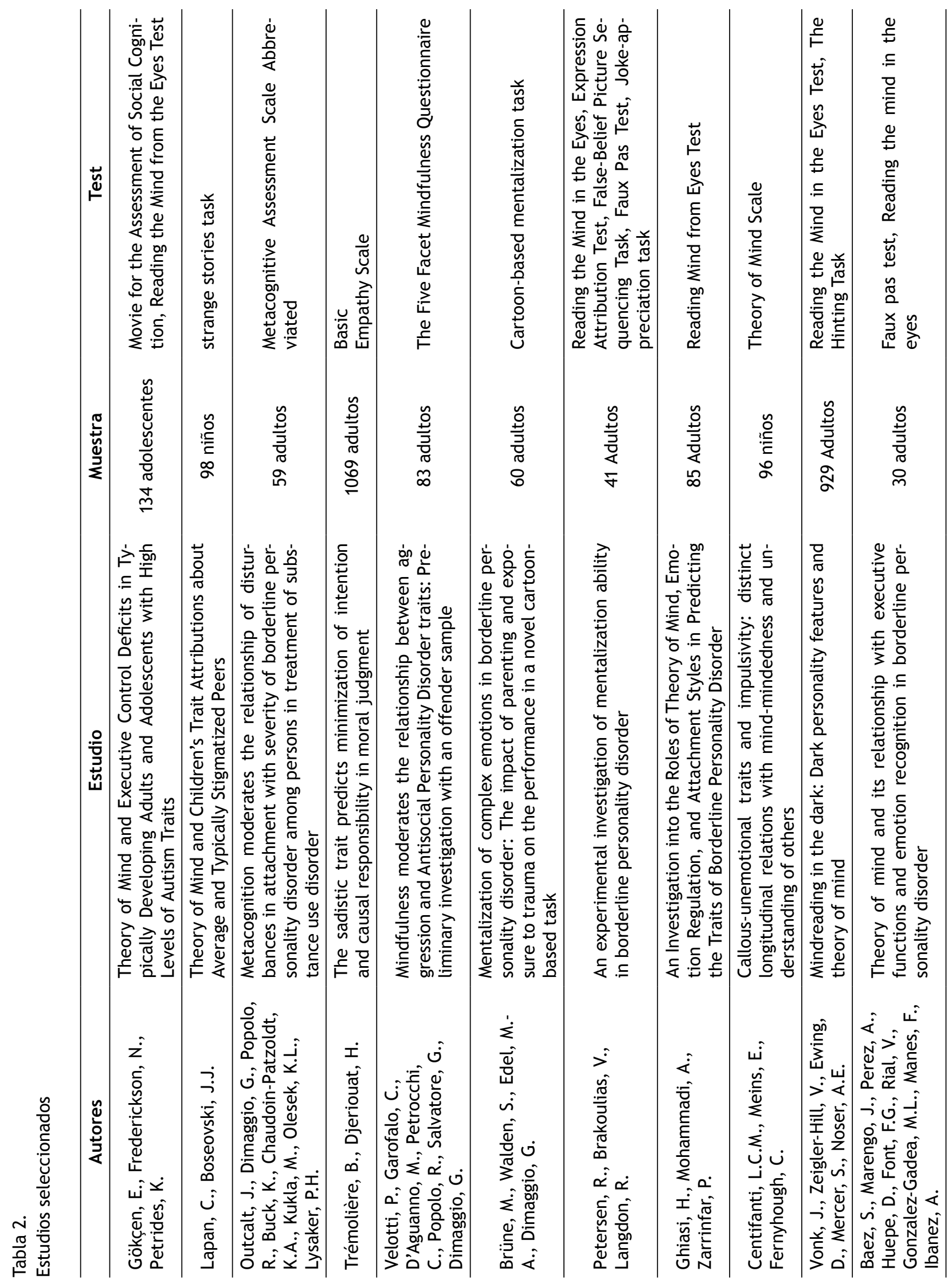




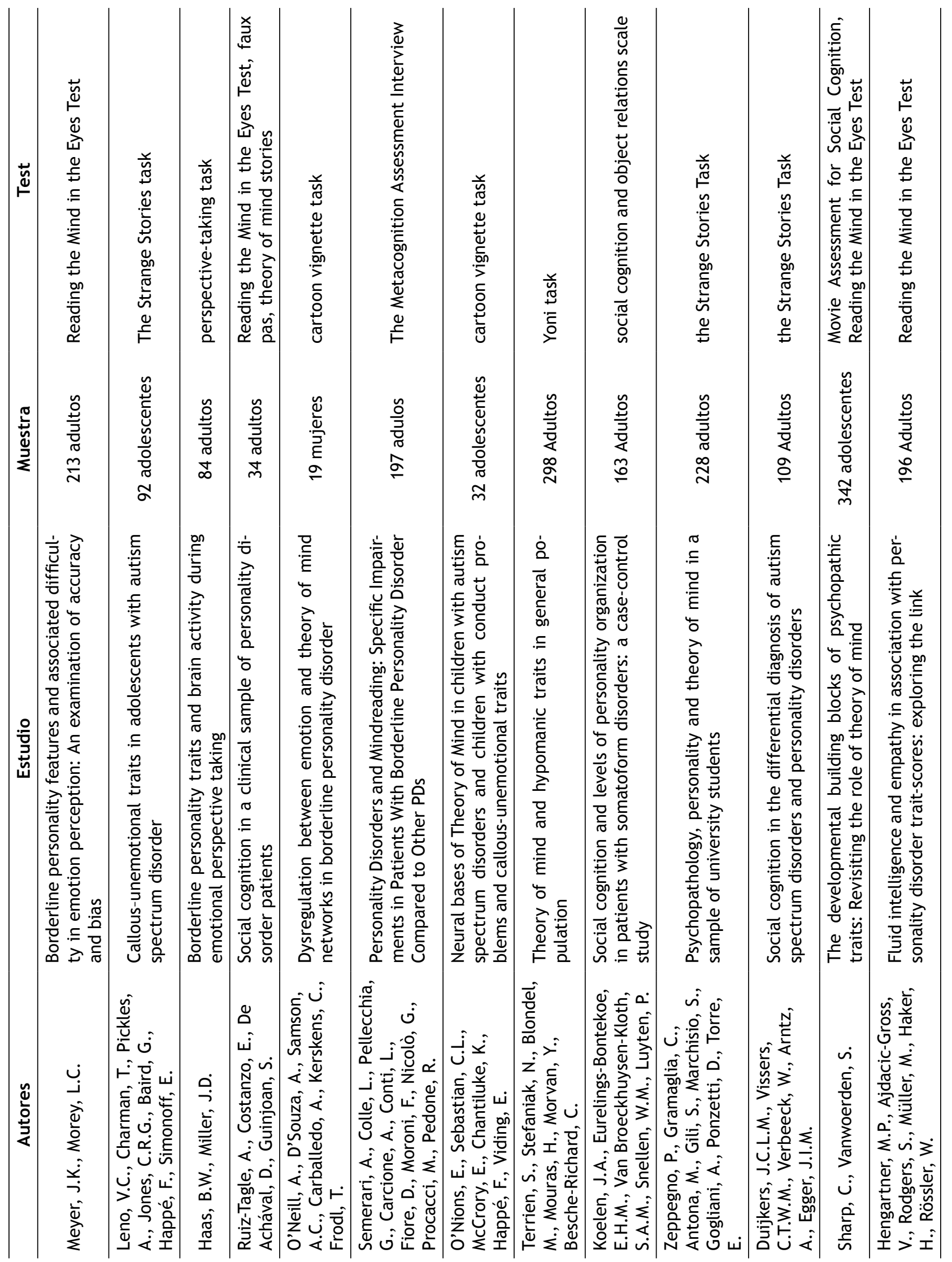




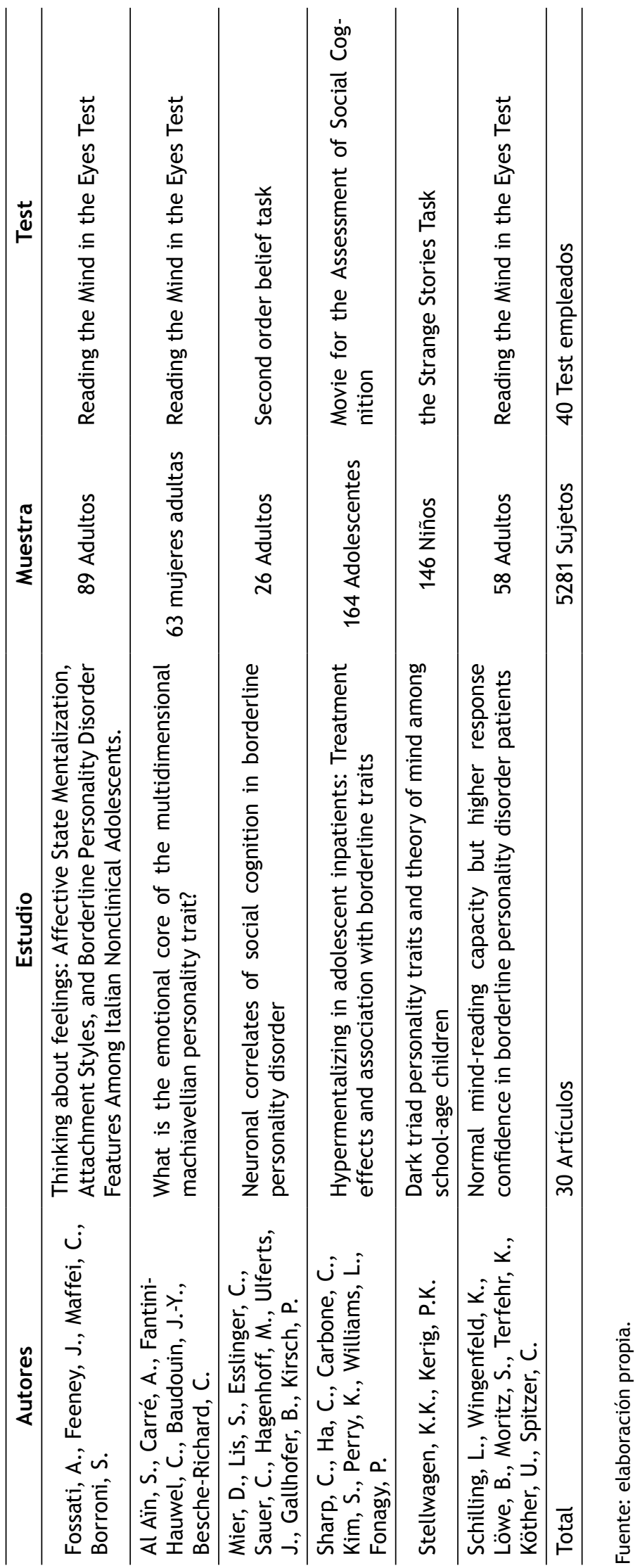


En los estudios seleccionados se realizó una categorización por año y por país de publicación, encontrando que el año con más publicaciones fue el 2016 con el 30\% de la muestra, seguido por los años 2015 y 2014 que presentan un porcentaje de $26.67 \%$ de los estudios (ver tabla 3). En lo referente al país de publicación se encontró que Estados Unidos presenta el mayor aporte de artículos con el 30\% de la muestra, seguido por Italia con el $13.33 \%$ y por Alemania, Reino Unido y Francia con el $10 \%$ cada uno de ellos (ver tabla 4).

Tabla 3.

Artículos producidos por año

\begin{tabular}{ccc}
\hline Año & Artículos & Porcentaje \\
\hline 2016 & 9 & 30.00 \\
2015 & 8 & 26.67 \\
2014 & 8 & 26.67 \\
2013 & 4 & 13.33 \\
2012 & 1 & 3.33 \\
\hline Total & 30 & 100 \\
\hline
\end{tabular}

Fuente: elaboración propia.

Tabla 4.

Artículos producidos por país

\begin{tabular}{lcc}
\hline País & Artículos & Porcentaje \\
\hline Estados Unidos & 9 & 30.00 \\
\hline Italia & 4 & 13.33 \\
Alemania & 3 & 10.00 \\
Reino Unido & 3 & 10.00 \\
Francia & 3 & 10.00 \\
Argentina & 2 & 6.67 \\
Holanda & 2 & 6.67 \\
Australia & 1 & 3.33 \\
Irán & 1 & 3.33 \\
Irlanda & 1 & 3.33 \\
Suiza & 1 & 3.33 \\
\hline Total & 30 & 100 \\
\hline
\end{tabular}

Fuente: elaboración propia.
En lo relacionado con las áreas temáticas de las revistas de publicación en los artículos seleccionados, se encontró que la mayoría de publicaciones acerca de las variables de ToM y personalidad se realizan en revistas de Medicina $76.67 \%$. Psicología $60.00 \%$ y Neurociencia $26.67 \%$, es importante en este punto mencionar que se encontraron revistas que trabajan diversos ejes temáticos de publicación, por lo cual la sumatoria de los porcentajes se vio alterada en los resultados (ver tabla 5).

Tabla 5.

Áreas temáticas de artículos seleccionados

\begin{tabular}{lcc}
\hline Área temática & Artículos & Porcentaje \\
\hline Medicina & 23 & 76.67 \\
Psicología & 18 & 60.00 \\
Neurociencia & 8 & 26.67 \\
Artes y Humanidades & 1 & 3.33 \\
Enfermería & 1 & 3.33 \\
Ciencias Sociales & 1 & 3.33 \\
\hline Total & 30 & 100 \\
\hline
\end{tabular}

Fuente: elaboración propia.

Por otro lado, en cuanto a las variables trabajadas y medidas en los artículos, se encontró que además de la medición de la ToM en relación a la personalidad, algunas otras variables también fueron medidas simultáneamente, entre ellas destacan: el Trastorno límite de personalidad en el $36.67 \%$, los rasgos alexitímicos en el $20 \%$, los correlatos neuronales de ambas variables en el $20 \%$ y los rasgos psicopáticos y el trastorno antisocial de la personalidad en el $20 \%$ de los estudios (ver tabla 6 ). 
Tabla 6.

Variables medidas en los artículos seleccionados

\begin{tabular}{lcc}
\hline Variable medida & Artículos & Porcentaje \\
\hline Teoría de la mente & 30 & 100 \\
Personalidad & 30 & 100 \\
Trastorno límite de personalidad & 11 & 36.67 \\
Rasgos alexitímicos & 6 & 20.00 \\
Correlatos neuronales & 6 & 20.00 \\
Rasgos psicopáticos y trastorno antisocial de la personalidad & 6 & 20.00 \\
Autismo & 5 & 16.67 \\
Control ejecutivo & 5 & 16.67 \\
Trastornos de personalidad & 5 & 16.67 \\
Apego & 4 & 13.33 \\
Empatía & 4 & 13.33 \\
Agresividad & 2 & 6.67 \\
Anhedonia & 1 & 3.33 \\
Ansiedad & 1 & 3.33 \\
Regulación emocional & 1 & 3.33 \\
Exposición a eventos traumáticos & 1 & 3.33 \\
Inteligencia & 1 & 3.33 \\
Juicio Moral & 1 & 3.33 \\
Trastorno narcisista de personalidad & 1 & 3.33 \\
Parentalidad & 1 & 3.33 \\
Trastornos somatomorfos & 1 & 3.33 \\
Abuso de sustancias & 1 & 3.33 \\
\hline
\end{tabular}

Fuente: elaboración propia.

Para la medición de la ToM fueron empleados distintos instrumentos, destacando el Reading the Mind from the Eyes Test con un 40\%, el Strange
Stories Task con $16.67 \%$ y el Cartoon Vignette Task, Faux Pas Test y el Movie for the Assessment of Social Cognition con un $10 \%$ cada uno (ver tabla 7).

Tabla 7.

Test empleados para la medición

\begin{tabular}{lcc}
\hline \multicolumn{1}{c}{ Test } & Artículos & Porcentaje \\
\hline Reading the Mind from the Eyes Test & 12 & 40.00 \\
Strange Stories Task & 5 & 16.67 \\
Cartoon Vignette Task & 3 & 10.00 \\
Faux Pas Test & 3 & 10.00 \\
Movie for the Assessment of Social Cognition & 3 & 10.00 \\
Empathy Scale & 1 & 3.33 \\
Expression Attribution Test & 1 & 3.33 \\
False-Belief Picture Sequencing Task & 1 & 3.33 \\
\hline
\end{tabular}




\begin{tabular}{lcc}
\hline \multicolumn{1}{c}{ Test } & Artículos & Porcentaje \\
\hline Metacognitive Assessment Scale Abbreviated & 1 & 3.33 \\
Joke-Appreciation Task & 1 & 3.33 \\
Perspective-Taking Task & 1 & 3.33 \\
Second order belief experimental task & 1 & 3.33 \\
social cognition and object relations scale & 1 & 3.33 \\
The Hinting Task & 1 & 3.33 \\
The Metacognition Assessment Interview & 1 & 3.33 \\
Theory of Mind Scale & 1 & 3.33 \\
Theory Of Mind Stories & 1 & 3.33 \\
The Situational Test of Emotion Management & 1 & 3.33 \\
The Situational Test of Emotion Understanding & 1 & 3.33 \\
Yoni task & 1 & 3.33 \\
\hline
\end{tabular}

Fuente: elaboración propia.

\section{Discusión}

Queda claro que el principal objetivo en los artículos fue estudiar posibles relaciones de la ToM con los trastornos de personalidad, y con rasgos disfuncionales que estuviesen relacionados con la impulsividad y con las conductas de daño o de exclusión social hacia los demás. En los estudios se confirmó la relación de deterioro de la ToM en personas con trastornos de personalidad (Ruiz-Tagle et al., 2015), en especial con el trastorno Límite, antisocial, narcisista, esquizoide y evitativo (Hengartner et al., 2013). Frente a la impulsividad también se obtuvieron hallazgos, encontrando relación entre la ToM y las conductas agresivas (Centifanti et al., 2015; Sharp \& Vanwoerden, 2014; Stellwagen \& Kerig, 2013; Velotti et al., 2016). En lo referente a la exclusión social, pudo hallarse una relación entre ambas variables donde se menciona que menores puntuaciones de ToM muestran mayores fenómenos de exclusión social inclusive desde etapas tempranas del desarrollo (Lapan \& Boseovski, 2015).

En lo cuanto al trastorno límite se encontró que existen posturas encontradas en relación con la existencia de disfunción en la ToM, mientras algunos estudios indican una clara disfunción en este dominio (Baez et al., 2014; Brüne et al., 2016; Ghiasi et al., 2016; Hengartner et al., 2013; Meyer \& Morey, 2015; Semerari et al., 2015; Sharp et al., 2013; Outcalt et al., 2016; Petersen et al., 2016), otros mencionan no encontrar diferencias con los grupos control (Fossati et al., 2014; Mier et al., 2012; Schilling et al., 2012). Estos resultados parecen reflejar que existe un enfoque emocional diferencial en el procesamiento de estímulos sociales en el trastorno límite aunque demuestren en ocasiones un buen desempeño en las tareas social-cognitivas estandarizadas, porque la base de los déficits sociocognitivos se evidencia en las interacciones sociales de la vida real (Mier et al., 2012). Con esto, se propone la implementación de test que involucren escenarios sociales más realistas para la medición de la ToM, y no sólo se enfoquen en medir si existe o no alteración del proceso (Baez et al., 2014). Desde este punto, puede comprenderse que los errores que se cometen en las interpretaciones sociales en el trastorno límite se presenta por problemas de sobre-interpretación de las situaciones y no por bajo entendimiento de las situaciones sociales (Sharp et al., 2013), aspecto que no es tenido en cuenta en todos los instrumentos empleados para la medición de la ToM.

En lo referente a la actividad de estructuras cerebrales y su relación con los rasgos de personalidad, se encontraron diferencias de activación en tareas relacionadas con la ToM. Los pacientes con trastorno límite de personalidad, mostraron una disminución significativa de la conectividad funcional en la corteza cingulada anterior y tres áreas cerebrales involucradas en los procesos de ToM: el lóbulo 
temporal superior izquierdo, los lóbulos parietales supramarginal derecho / inferior y el córtex cingulado medio derecho. Además de diferencias de activación en distintas estructuras como el precuneus y el lóbulo frontal inferior izquierdo, izquierdo precentral / medio frontal y izquierdo medio occipital / parietal superior lóbulos en reposo (O’Neill et al., 2015). También se evidenció una hipoactivación en dos regiones cerebrales involucradas en la empatía cognitiva, el surco temporal superior y la unión temporo-parietal (Haas \& Miller, 2015), e hiperactivación en la amígdala. Esto, lleva a concluir que este patrón de activación parece reflejar un enfoque emocional diferencial en el procesamiento de estímulos sociales en personas con rasgos límites (Mier et al., 2012), Además de proponer que el aumento de la conectividad entre el precuneus y las regiones frontales podría estar relacionado con el procesamiento extensivo de pensamientos y la posterior información autorreferencial en los pacientes límites (O’Neill et al., 2015).

Algunos de los estudios presentaron además análisis de funciones ejecutivas con relación a las dos variables principales. En estos estudios los hallazgos apoyan la noción de que la función ejecutiva y la teoría de la mente son habilidades relacionadas (Baez et al., 2014; Gökçen, Frederickson, \& Petrides, 2016), entre estas se encontró influencia de la memoria de trabajo (Lapan \& Boseovski, 2015) y el control inhibitorio (Lapan \& Boseovski, 2015; Meyer \& Morey, 2015).

Otro aspecto relevante fue la inclusión de las variables de tipo de apego en relación con la ToM. En esta temática se halló que este dominio metacognitivo moderaba la relación entre el estilo de apego disfuncional con la cronicidad de rasgos de personalidad limítrofes (Ghiasi et al., 2016). En estos estudios se evidenció que a mayores niveles de apego ansioso y de menores puntuaciones de la ToM se presentaran mayor cronicidad del trastorno de personalidad (Outcalt et al., 2016; Semerari et al., 2015; Zeppegno, et al., 2014). Además de identificar, más bajas las puntuaciones de ToM a mayor presencia de experiencias infantiles de castigo (Petersen et al., 2016), y a mayor número de experiencias traumáticas y a menor calidad de vinculo parental durante la niñez (Brüne et al.,2016).
Las variables insensibilidad emocional y alexitímia también demuestra gran interés en los estudios de la actual revisión. Desde este punto, se propone que las características de insensibilidad emocional manifestadas tanto por Autismo, como por rasgos de personalidad al analizarlas desde la perspectiva de bajos niveles de ToM, pueden llevar a justificar la presencia del grado de disfunción social y ejecutiva que presentan ambas categorías diagnósticas (Duijkers, et al., 2014; Gökçen et al., 2016; O’Nions et al., 2014; Stellwagen \& Kerig, 2013), inclusive se propone que es posible desde los 10 años de edad con test de la ToM predecir la presencia de rasgos de insensibilidad emocional futuros (Centifanti, et al., 2015). La insensibilidad emocional además de asociarse con la ToM, lo ha hecho con la dificultad para el reconocimiento del miedo en los demás, lo cual a su vez mostró relación con la presencia de problemas de conducta en la población no clínica (Leno et al., 2015)

El Trastorno antisocial y la psicopatía son otras dos variables relevantes en los artículos rastreados. En estos se encontró una relación entre las puntuaciones de la ToM y el trastorno antisocial (Al Ain et al., 2013; Velotti et al., 2016), así como con características psicopáticas (Vonk et al., 2015) y rasgos de sadismo (Al Aïn, et al., 2013; Trémolière \& Djeriouat, 2016). De igual manera, la ToM demostró que en bajas puntuaciones puede relacionarse con los niveles de agresividad en los sujetos con esta condición (Sharp \& Vanwoerden, 2014; Velotti et al., 2016), pero de forma paradójica en los sujetos con tendencias más cercanas a la psicopatía, donde demostraban presencia de rasgos de grandiosidad y narcisismo las puntuaciones en la ToM demostraban ser más elevadas que las del grupo control (Sharp \& Vanwoerden, 2014; Vonk, et al., 2015). Estos hallazgos sugieren que los individuos con algunas características de personalidad antisocial pueden leer mal los estados mentales, llevándolos a actuar de forma impulsiva, aquellos que demuestran psicopatía pueden presentar puntuaciones más altas que pueden ayudarle en las conductas de manipulación típicas en estos individuos (Vonk et al., 2015).

La capacidad empática también demuestra de forma reiterativa su relevancia de estudio en función de la ToM. En varios de los estudios se concluyó 
que distintos tipos de organización disfuncional de la personalidad, presentaban conjuntamente disfunción de la ToM, lo cual a su vez, les permitió relacionar los bajos niveles empáticos con las bajas puntuaciones de la ToM (Al Aïn, et al., 2013; Hengartner et al., 2013; Petersen et al., 2016; Sharp \& Vanwoerden, 2014).

Por último, en otras variables también se encontraron hallazgos relevantes en relación con la baja ToM, como encontrar distorsionada la capacidad y juicio moral en sujetos sádicos (Trémolière \& Djeriouat, 2016), por aumentos de rasgos de personalidad similares a los hipomaniacos (Terrien et al., 2013), rasgos de personalidad que favorecían un mejor desempeño académico (Zeppegno et al., 2014).

\section{Conclusiones}

En la totalidad de los estudios, la ToM es considerada como una variable que se relaciona con el adecuado desempeño social por la función interpretativa que se le atribuye. Con respecto a la personalidad se identifica una relación en bajas puntuaciones con estructuras patológicas como lo son los trastornos de la personalidad, más claramente con estructuras limítrofes, antisociales y narcisistas; de igual mane$\mathrm{ra}$, en altos niveles también muestra relación con los rasgos psicopáticos de personalidad. Además, se refiere que la sintomatología propia de estos trastornos tiende a agravarse cuando se presenta mayor disfunción en la ToM.

El tipo de apego tiene un nexo de relación con la ToM. Se identifica que, a mayores experiencias adversas en la niñez, mayor disfunción es adquirida en el estilo vincular y en las interpretaciones adecuadas que realiza en los ámbitos sociales. Las experiencias más relacionadas son traumas, crianzas punitivas y menor calidad de vinculo parental.

Los diversos tipos de instrumentos para evaluar la ToM muestran en su mayoría la tendencia a medir únicamente aciertos y errores en la interpretación de los estados mentales, aspecto que no permite identificar el motivo etiológico del error. Así mismo, algunos de los nuevos test audiovisuales permiten identificar el motivo del error en el evaluado, y muestran mayor posibilidad de acercamiento a los ambientes sociales reales donde se desenvuelve el sujeto.

Otros aspectos que se derivan de la personalidad como la empatía, la insensibilidad emocional, el juicio moral, el rendimiento académico y comportamientos cercanos a rasgos hipomaniacos, son variables que demuestra afección en relación con las puntaciones bajas de la ToM, especialmente en personas con estructuras disfuncionales de personalidad.

Aunque se ha avanzado en el conocimiento de las alteraciones de la ToM en relación a la personalidad, las investigaciones no son lo suficientemente extensas para aclarar cómo opera esta interacción con otras estructuras de personalidad. Además, no se encuentran investigaciones realizadas en la multiplicidad de dimensiones y de rasgos de personalidad en sujetos sin trastorno, aspecto que de investigarse permitiría establecer nuevas explicaciones de la influencia de este aspecto metacognitivo en el desarrollo de los rasgos de personalidad y en las posteriores disfunciones comportamentales que pueden presentarse también en sujetos sin psicopatología.

\section{Limitaciones del estudio}

En esta revisión no fue posible encontrar revisiones meta-analíticas que pudiesen establecer una relación entre la ToM y los rasgos o estructuras de personalidad. En las investigaciones examinadas el mayor alcance fue el de establecer correlaciones y puntajes de varianza para la comprensión e influencia de las variables de estudio. No es del alcance de esta investigación permitir hacer interpretaciones causales o relacionales acerca de la ToM con la personalidad, para realizar esto es necesario realizar otros estudios con distintos alcances investigativos que permitan realizar este tipo de inferencias. 


\section{Referencias}

Adachi, T., Koeda, T., Hirabayashi, S., Maeoka, Y., Shiota, M., ... Wada, A. (2004). The metaphor and sarcasm scenario test: a new instrument to help differentiate high functioning pervasive developmental disorder from attention deficit/ hyperactivity disorder. Brain and Development, 26(5), 301-306.

Adenzato, M., Cavallo, M., \& Enrici, I. (2010). Theory of Mind ability in the behavioral variant of frontotemporal dementia: An analysis of the neural, cognitive, and social levels. Neuropsychologia, 48(1), 2-12.

Adolphs, R., Tranel, D., Damasio, H., \& Damasio, A. (1994). Impaired recognition of emotion in facial expressions following bilateral damage to the human amygdala. Neuropsychologia, 372, 669-672.

*Al Aïn, S., Carré, A., Fantini-Hauwel, C., Baudouin, J., \& Besche-Richard, C. (2013). What is the emotional core of the multidimensional Machiavellian personality trait? Frontiers in Psychology, 4, 1-8.

*Baez, S., Marengo, J., Perez, A., Huepe, D., Font, F., ... Ibañez, A. (2014). Theory of mind and its relationship with executive functions and emotion recognition in borderline personality disorder. Journal of Neuropsychology, 9(2), 203-218.

Baker, C., Peterson, E., Pulos, S., \& Kirkland, R. (2014). Eyes and IQ: A meta-analysis of the relationship between intelligence and "Reading the Mind in the Eyes". Intelligence, 44, 78-92. DOI: 10.1016/j.intell.2014.03.001

Baron-Cohen, S. (1989). The Autistic Child's Theory of Mind: A Case of Specific Developmental Delay. Journal of Child Psychology and Psychiatry, 30(2), 285-297.

Baron-Cohen, S., Jolliffe, T., Mortimore, C., \& Robertson, M. (1997). Another Advanced Test of Theory of Mind: Evidence from Very High Functioning Adults with Autism or Asperger Syndrome. Journal of Child Psychology and Psychiatry, 38(7), 813-822.
Baron-Cohen, S., Leslie, A., \& Frith, U. (1985). Does the autistic child have a "Theory of Mind"? Cognition, 21(1), 37-46.

Baron-Cohen, S., O'Riordan, M., Stone, V., Jones, R., \& Plaisted, K. (1999). Recognition of Faux Pas by Normally Developing Children and Children with Asperger Syndrome or High-Functioning Autism. Journal of Autism Development Disordorders, 29(5), 407-418.

Blijd-Hoogewys, E., van Geert, P., Serra, M., \& Minderaa, R. (2008). Measuring Theory of Mind in Children. Psychometric Properties of the ToM Storybooks. Journal of Autism and Developmental Disorders, 38(10), 1907-1930.

Bliksted, V., Ubukata, S., \& Koelkebeck, K. (2016). Discriminating autism spectrum disorders from schizophrenia by investigation of mental state attribution on an on-line mentalizing task: A review and meta-analysis. Schizophrenia Research, 171(1-3), 16-26.

Bora, E., Bartholomeusz, C., \& Pantelis, C. (2015). Meta-analysis of Theory of Mind (ToM) impairment in bipolar disorder. Psychological Medicine, 46(02), 253-264.

Bora, E., \& Pantelis, C. (2013). Theory of mind impairments in first-episode psychosis, individuals at ultra-high risk for psychosis and in firstdegree relatives of schizophrenia: Systematic review and meta-analysis. Schizophrenia Research, 144(1-3), 31-36.

Bora, E. \& Pantelis, C. (2015). Meta-analysis of social cognition in attention-deficit/hyperactivity disorder (ADHD): comparison with healthy controls and autistic spectrum disorder. Psychological Medicine, 46(04), 699-716. DOI: 10.1017/s0033291715002573.

Bora, E., Velakoulis, D., \& Walterfang, M. (2016). Social cognition in Huntington's disease: A meta-analysis. Behavioural Brain Research, 297, 131-140.

Bora, E., Yucel, M., \& Pantelis, C. (2009). Theory of mind impairment in schizophrenia: Metaanalysis. Schizophrenia Research, 109(1-3), 1-9. 
Bora, E., Walterfang, M., \& Velakoulis, D. (2015). Theory of mind in Parkinson's disease: A metaanalysis. Behavioural Brain Research, 292, 515520. DOI: 10.1016/j.bbr.2015.07.012

Bretherton, I. \& Beeghly, M. (1982). Talking about internal states: The acquisition of an explicit Theory of Mind. Developmental Psychology, 18(6), 906-921.

Broks, P., Young, A., Maratos, E., Coffey, P., Callder, A., ... Hadley, D. (1998). Face processing impairments after encephalitis: Amygdala damage and recognition of fear. Neuropsychologia, 36, 59-70.

Brüne, M. (2005). Emotion recognition, 'Theory of Mind' and social behaviour in Schizophrenia. Psychiatry Research, 133, 135-147. DOI: 10.1016/j.psychres.2004.10.007.

*Brüne, M., Walden, S., Edel, M., \& Dimaggio, G. (2016). Mentalization of complex emotions in borderline personality disorder: The impact of parenting and exposure to trauma on the performance in a novel cartoon-based task. Comprehensive Psychiatry, 64, 29-37.

${ }^{*}$ Centifanti, L., Meins, E., \& Fernyhough, C. (2015). Callous-unemotional traits and impulsivity: distinct longitudinal relations with mind-mindedness and understanding of others. Journal of Child Psychology and Psychiatry, 57(1), 84-92.

Corcoran, R., Cahill, C., \& Frith, C. D. (1997). The appreciation of visual jokes in people with schizophrenia: A study of "mentalizing" ability. Schizophrenia Research, 24, 319-327.

Corcoran, R., Mercer, G., \& Frith, C. (1995). Schizophrenia, symptomatology and social inference: Investigating "theory of mind" in people with schizophrenia. Schizophrenia Research, 17(1), 5-13.

Deptula, A. \& Bedwell, J. (2015). Schizotypy, Autobiographical Memory, and Theory of Mind. The Journal of Nervous and Mental Disease, 203(2), 96-100.
*Duijkers, J. C. L. M., Vissers, C. Th. W. M., Verbeeck, W., Arntz, A. \& Egger, J. I. M. (2014). Social cognition in the differential diagnosis of autism spectrum disorders and personality disorders. Clinical Neuropsychiatry, 11(5), 118-129.

Dziobek, I., Fleck, S., Kalbe, E., Rogers, K., Hassenstab, J., \& Brand, M. et al.. (2006). Introducing MASC: A Movie for the Assessment of Social Cognition. Journal Of Autism And Developmental Disorders, 36(5), 623-636. DOI: 10.1007/s10803-006-0107-0

Emery, N. \& Amaral, D. (2000). The role of the amygdala in primate social cognition. En R. Lane \& L. Nadel (Eds.), Cognitive Neuroscience of Emotion. Oxford: Oxford University Press.

Fett, A., Viechtbauer, W., Dominguez, M., Penn, D., van Os, J., \& Krabbendam, L. (2011). The relationship between neurocognition and social cognition with functional outcomes in schizophrenia: A meta-analysis. Neuroscience \& Biobehavioral Reviews, 35(3), 573-588.

*Fossati, A., Feeney, J., Maffei, C., \& Borroni, S. (2014). Thinking about feelings: Affective state mentalization, attachment styles, and borderline personality disorder features among Italian nonclinical adolescents. Psychoanalytic Psychology, 31(1), 41-67.

García, E. (2008). Neuropsicología y Educación: De las neuronas espejo a la teoría de la mente. Revista de Psicología y Educación, 1(3), 69-90.

*Ghiasi, H., Mohammadi, A., \& Zarrinfar, P. (2016). An Investigation into the Roles of Theory of Mind, Emotion Regulation, and Attachment Styles in Predicting the Traits of Borderline Personality Disorder. Iranian Journal of Psychiatry, 11(4), 206-213.

Gökçen, E., Frederickson, N., \& Petrides, K. (2016). Theory of Mind and Executive Control Deficits in Typically Developing Adults and Adolescents with High Levels of Autism Traits. Journal of Autism and Developmental Disorders, 46(6), 2072-2087. 
*Haas, B. \& Miller, J. (2015). Borderline personality traits and brain activity during emotional perspective taking. Personality Disorders: Theory, Research, and Treatment, 6(4), 315-320.

Happé, F. (1993). Communicative competence and theory of mind in autism: A test of relevance theory. Cognition, 48(2), 101-119.

Happé, F. (1994). An advanced test of Theory of Mind: Understanding of story characters' thoughts and feelings by able autistic, mentally handicapped, and normal children and adults. Journal of Autism and Developmental Disorders, 24(2), 129-154.

Heavey, L., Phillips, W., Baron-Cohen, S., \& Rutter, M. (2000). The Awkward Moments Test: a naturalistic measure of social understanding in autism. Journal of Autism and Developmental Disorders, 30(3), 225-236.

*Hengartner, M., Ajdacic-Gross, V., Rodgers, S., Müller, M., ... Rössler, W. (2013). Fluid intelligence and empathy in association with personality disorder trait-scores: exploring the link. European Archives of Psychiatry and Clinical Neuroscience, 264(5), 441-448.

Henry, J., Bailey, P., \& Rendell, P. (2008). Empathy, social functioning and schizotypy. Psychiatry Research, 160(1), 15-22. DOI: 10.1016/j. psychres.2007.04.014

Hood, L., Bloom, L., \& Brainerd, C. (1979). What, When, and How about Why: A Longitudinal Study of Early Expressions of Causality. Monographs of The Society for Research in Child Development, 44(6), 1-47.

Kaland, N., Moller-Nielsen, A., Callesen, K., Mortensen, E., Gottlieb, D., \& Smith, L. (2002). A new 'advanced' test of theory of mind: evidence from children and adolescents with Asperger syndrome. Journal of Child Psychology and Psychiatry, 43(4), 517-528.

*Koelen, J., Eurelings-Bontekoe, E., van Broeckhuysen-Kloth, S., Snellen, W., \& Luyten, P. (2014). Social Cognition and Levels of Personality Organization in Patients With Somatoform Disorders. The Journal of Nervous and Mental Disease, 202(3), 217-223.

Korkman, M., Kirk, U., \& Kemp, S. (2007). NEPSYII: Administration manual. San Antonio: Psychological Corporation.

Lamm C. \& Singer T. (2010). The role of anterior insular cortex in social emotions. Brain Structure \& Function, 214, 579-591.

*Lapan, C. \& Boseovski, J. (2015). Theory of Mind and Children's Trait Attributions about Average and Typically Stigmatized Peers. Infant and Child Development, 25(2), 158-178.

Lavoie, M., Plana, I., Bédard Lacroix, J., GodmaireDuhaime, F., Jackson, P., \& Achim, A. (2013). Social cognition in first-degree relatives of people with Schizophrenia: A meta-analysis. Psychiatry Research, 209(2), 129-135.

*Leno, V., Charman, T., Pickles, A., Jones, C., Baird, G., ... Simonoff, E. (2015). Callous-unemotional traits in adolescents with autism spectrum disorder. The British Journal of Psychiatry, 207(5), 392-399.

Marchetti, A., Baglio, F., Costantini, I., Dipasquale, O., Savazzi, F., ... Castelli, I. (2015). Theory of Mind and the Whole Brain Functional Connectivity: Behavioral and Neural Evidences with the Amsterdam Resting State Questionnaire. Frontiers in Psychology, 6, 1-10.

Martins-Junior, F., Sanvicente-Vieira, B., GrassiOliveira, R., \& Brietzke, E. (2011). Social cognition and Theory of Mind: Controversies and promises for understanding major psychiatric disorders. Psychology \& Neuroscience, 4(3), 347-351. DOI: 10.3922/j.psns.2011.3.008

*Meyer, J. \& Morey, L. (2015). Borderline personality features and associated difficulty in emotion perception: An examination of accuracy and bias. Personality and Mental Health, 9(3), 227-240. 
*Mier, D., Lis, S., Esslinger, C., Sauer, C., Hagenhoff, M., ... Kirsch, P. (2012). Neuronal correlates of social cognition in borderline personality disorder. Social Cognitive and Affective Neuroscience, 8(5), 531-537.

Muris, P., Steerneman, P., Meesters, C., Merckelbach, H., Horselenberg, R., van den Hogen, T. \& van Dongen, L. (1999). The TOM Test: A new instrument for assessing Theory of Mind in normal children and children with pervasive developmental disorders. Journal of Autism and Developmental Disorders, 29(1), 67-78.

Nentjes, L., Bernstein, D., Arntz, A., Slaats, M., \& Hannemann, T. (2015). Theory of Mind, Social Desirability, and Unlikely Symptom Reporting in Offenders With and Without Psychopathy. The Journal of Nervous and Mental Disease, 203(8), 596-603.

Nentjes, L., Bernstein, D., Arntz, A., van Breukelen, G., \& Slaats, M. (2015). Examining the influence of psychopathy, hostility biases, and automatic processing on criminal offenders' Theory of Mind. International Journal of Law and Psychiatry, 38, 92-99.

*O'Neill, A., D'Souza, A., Samson, A., Carballedo, A., Kerskens, C., \& Frodl, T. (2015). Dysregulation between emotion and theory of mind networks in borderline personality disorder. Psychiatry Research: Neuroimaging, 231(1), 25-32.

*O'Nions, E., Sebastian, C., McCrory, E., Chantiluke, K., Happé, F., \& Viding, E. (2014). Neural bases of Theory of Mind in children with autism spectrum disorders and children with conduct problems and callous-unemotional traits. Developmental Science, 17(5), 786-796.

*Outcalt, J., Dimaggio, G., Popolo, R., Buck, K., Chaudoin-Patzoldt, K., ... Lysaker, P. H. (2016). Metacognition moderates the relationship of disturbances in attachment with severity of borderline personality disorder among persons in treatment of substance use disorders. Comprehensive Psychiatry, 64, 22-28.

*Petersen, R., Brakoulias, V., \& Langdon, R. (2016). An experimental investigation of mentaliza- tion ability in borderline personality disorder. Comprehensive Psychiatry, 64, 12-21.

Premack, D. \& Woodruff, G. (1978). Does the chimpanzee have a theory of mind? Behavioral and Brain Sciences, 1(04), 515.

Roeyers, H., Buysse, A., Ponnet, K., \& Pichal, B. (2001). Advancing Advanced Mind-reading Tests: Empathic Accuracy in Adults with a Pervasive Developmental Disorder. Journal of Child Psychology and Psychiatry, 42(2), 271-278.

*Ruiz-Tagle, A., Costanzo, E., De Achával, D., \& Guinjoan, S. (2015). Social Cognition in a Clinical Sample of Personality Disorder Patients. Frontiers in Psychiatry, 6, 1-5.

Samamé, C., Martino, D., \& Strejilevich, S. (2011). Social cognition in euthymic bipolar disorder: systematic review and meta-analytic approach. Acta Psychiatrica Scandinavica, 125(4), 266-280.

Savla, G., Vella, L., Armstrong, C., Penn, D., \& Twamley, E. (2012). Deficits in Domains of Social Cognition in Schizophrenia: A Meta-Analysis of the Empirical Evidence. Schizophrenia Bulletin, 39(5), 979-992.

Schenkel, L. S., Spaulding, W. D. \& Silverstein, S. M. (2005). Poor premorbid social functioning and theory or mind deficit in Schizophrenia: evidence of reduced context processing. Journal of Psychiatric Research, 39, 499-508. DOI: 10.1016/j.jpsychires.2005.01.001.

*Schilling, L., Wingenfeld, K., Löwe, B., Moritz, S., Terfehr, K., ... Spitzer, C. (2012). Normal mind-reading capacity but higher response confidence in borderline personality disorder patients. Psychiatry and Clinical Neurosciences, 66(4), 322-327.

*Semerari, A., Colle, L., Pellecchia, G., Carcione, A., Conti, L., ... Pedone, R. (2015). Personality Disorders and Mindreading. The Journal of Nervous and Mental Disease, 203(8), 626-631.

*Sharp, C. \& Vanwoerden, S. (2014). Social Cognition: Empirical Contribution: The Developmental Building Blocks of Psychopathic 
Traits: Revisiting the Role of Theory of Mind. Journal of Personality Disorders, 28(1), 78-95.

*Sharp, C., Ha, C., Carbone, C., Kim, S., Perry, K., ... Fonagy, P. (2013). Hypermentalizing in Adolescent Inpatients: Treatment Effects and Association With Borderline Traits. Journal of Personality Disorders, 27(1), 3-18.

Shultz, T., Wells, D., \& Sarda, M. (1980). Development of the ability to distinguish intended actions from mistakes, reflexes, and passive movements. British Journal of Social and Clinical Psychology, 19(4), 301-310.

*Stellwagen, K. \& Kerig, P. (2013). Dark triad personality traits and theory of mind among school-age children. Personality and Individual Differences, 54(1), 123-127.

Stone, V. E., Baron-Cohen, S., \& Knight, R. T. (1998). Frontal lobe contributions to Theory of Mind. Journal of Cognitive Neuroscience, 10, 640-656.

Téllez-Vargas, J. (2006). Teoría de la mente: evolución, ontogenia, neurobiología y psicopatología. Avances en Psiquiatría Biológica, 7, 6-27.

*Terrien, S., Blondel, M., Morvan, Y., \& BescheRichard, C. (2013). 2618 - Theory of mind and hypomanic personality in general population. European Psychiatry, 28, 694-699.

Trevisan, D., \& Birmingham, E. (2016). Are emotion recognition abilities related to everyday social functioning in ASD? A meta-analysis. Research In Autism Spectrum Disorders, 32, 24-42. DOI: 10.1016/j.rasd.2016.08.004

*Trémolière, B. \& Djeriouat, H. (2016). The sadistic trait predicts minimization of intention and causal responsibility in moral judgment. Cognition, 146, 158-171.

van Donkersgoed, R., Wunderink, L., Nieboer, R., Aleman, A., \& Pijnenborg, G. (2015). Social Cognition in Individuals at Ultra-High Risk for Psychosis: A Meta-Analysis. PLOS ONE, 10(10), 1-16.

*Velotti, P., Garofalo, C., D’Aguanno, M., Petrocchi, C., Popolo, R., ... Dimaggio, G. (2016). Mindfulness moderates the relationship between aggression and Antisocial Personality Disorder traits: Preliminary investigation with an offender sample. Comprehensive Psychiatry, 64, 38-45.

*Vonk, J., Zeigler-Hill, V., Ewing, D., Mercer, S., \& Noser, A. (2015). Mindreading in the dark: Dark personality features and theory of mind. Personality and Individual Differences, 87, 50-54.

Wellman, H. \& Liu, D. (2004). Scaling of Theory-ofMind Tasks. Child Development, 75(2), 523-541.

Wellman, H., Fang, F., Liu, D., Zhu, L., \& Liu, G. (2006). Scaling of Theory-of-Mind Understandings in Chinese Children. Psychological Science, 17(12), 1075-1081.

Wimmer, H. \& Perner, J. (1983). Beliefs about beliefs: Representation and constraining function of wrong beliefs in young children's understanding of deception. Cognition, 13(1), 103-128.

Yirmiya, N., Erel, O., Shaked, M., \& Solomonica-Levi, D. (1998). Meta-analysis comparing Theory of Mind abilities of individuals with Autism, individuals with Mental Retardation, and normally developing individuals. Psychological Bulletin, 124(3), 283-307.

Zaborszky L., Gaykema, R.P., Swanson, D.J. \& Cullinan, E. (1997). Cortical input to the basal forebrain. Neuroscience, 79, 1051-1078.

*Zeppegno, P., Gramaglia, C., Antona, M., Gili, S., Marchisio, S., ... Torre, E. (2014). Psychopathology, personality and theory of mind in a sample of university students. Rivista Di Psichiatria, 49(3), 132-139. 